\title{
Technè
}

La science au service de l'histoire de l'art et de la préservation des biens culturels

$44 \mid 2016$

Archives de l'humanité : les restes humains patrimonialisés

\section{Les restes humains au conservatoire d'anatomie de la faculté de médecine de Montpellier}

Human remains in the Conservatoire d'Anatomie de la Faculté de Médecine, Montpellier

\section{Caroline Ducourau}

\section{(2) OpenEdition Journals}

Édition électronique

URL : http://journals.openedition.org/techne/951

DOI : 10.4000/techne.951

ISSN : 2534-5168

\section{Éditeur}

C2RMF

\section{Édition imprimée}

Date de publication : 1 novembre 2016

Pagination : 22-28

ISBN : 978-2-7118-6339-6

ISSN : 1254-7867

\section{Référence électronique}

Caroline Ducourau, «Les restes humains au conservatoire d'anatomie de la faculté de médecine de Montpellier », Technè [En ligne], 44 | 2016, mis en ligne le 19 décembre 2019, consulté le 28 juillet 2020. URL : http://journals.openedition.org/techne/951 ; DOI : https://doi.org/10.4000/techne.951

\section{cc)}

La revue Technè. La science au service de l'histoire de l'art et de la préservation des biens culturels est mise à disposition selon les termes de la Licence Creative Commons Attribution - Pas d'Utilisation Commerciale - Pas de Modification 4.0 International. 


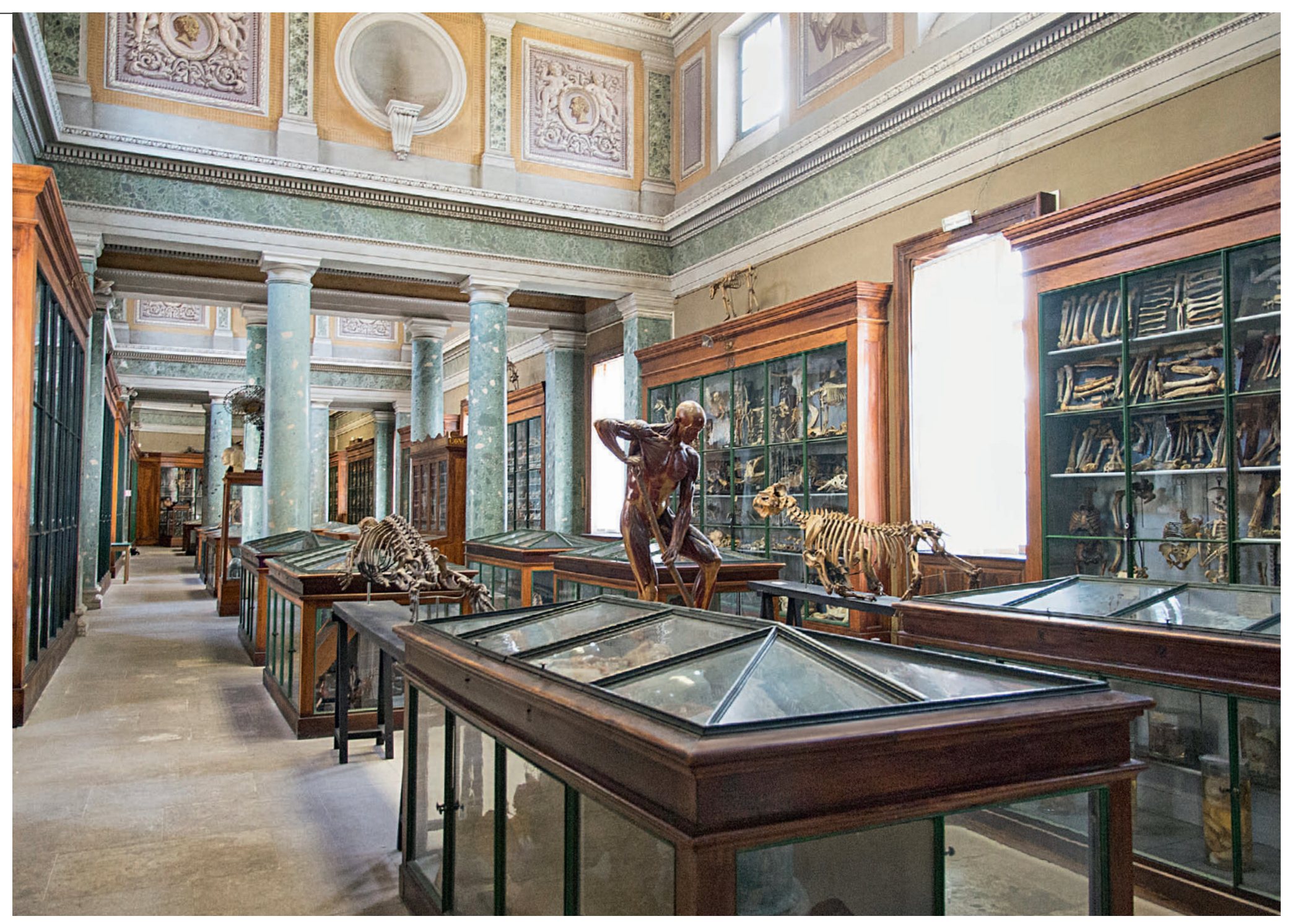

Fig. 1. Conservatoire d'anatomie, vue d'ensemble. ( ) D. Richard/Transit. 
Caroline Ducourau

\title{
Les restes humains au conservatoire d'anatomie de la faculté de médecine de Montpellier
}

\author{
Human remains in the Conservatoire \\ d'Anatomie de la Faculté de Médecine, \\ Montpellier
}

Résumé. La faculté de médecine de l'Université de Montpellier abrite une collection anatomique d'importance, présentée dans une galerie majestueuse construite à cet effet à la moitié du XIX $X^{e}$ siècle. L'origine de cette collection date des dernières années de la Révolution française, qui fut une période de profonde réforme de l'enseignement de la médecine et de la chirurgie, avec la création en 1794 de trois Écoles de Santé en France (Paris, Strasbourg et Montpellier), accompagnées chacune d'un conservatoire comprenant un cabinet d'anatomie destiné à l'enseignement pratique. La collection, qui est constituée quasiment pour une moitié de restes humains relatifs à l'anatomie humaine normale et pathologique, s'enrichit au cours des XIX ${ }^{e}$ et XX $X^{e}$ siècles par l'activité même de l'École pratique d'anatomie et de chirurgie, et grâce aux nombreux dons de professeurs ou de chirurgiens. Elle est ainsi un témoignage remarquable des méthodes d'enseignement de l'anatomie avant l'avènement moderne de «l'anatomie du vivant».

Mots-clés. Médecine, Montpellier, enseignement, anatomie.
Abstract. The University of Montpellier's Faculty of Medicine houses an impressive anatomy collection, displayed in a magnificent gallery specially built in the mid-19th century. The origin of this collection dates back to the last years of the French Revolution, a period of extensive reform in the teaching of medicine and surgery, with the founding of three Schools of Health (in Paris, Strasbourg and Montpellier), in 1794, each comprising an academy that included an anatomy collection intended for practical teaching purposes. Almost half of the collection is composed of body parts showing normal and pathological human anatomy. In the course of the 19th and 20th centuries, the collection continued to expand thanks to the Faculty of Medicine's activities in practical anatomy and surgery, and numerous donations made by professors and surgeons. It thus offers remarkable insight into the teaching of anatomy before the advent of "living anatomy".

Keywords. Medicine, Montpellier, teaching, anatomy.
L'étude de l'homme revêt une grande diversité d'approches, selon que celui-ci est considéré dans son acception d'être vivant, d'être social, ou encore d'être sain, malade ou blessé. Les disciplines scientifiques s'intéressant à l'homme se sont ainsi spécialisées à partir du XIX ${ }^{\mathrm{e}}$ siècle et recouvrent plusieurs champs d'étude : la zoologie et l'anatomie comparée, où l'homme est le dernier maillon de la grande chaîne de l'évolution, l'ethnologie ou encore l'anatomie normale et pathologique. Les collections universitaires qui sont liées à ces champs d'étude rassemblent de nombreux restes humains, dont le mode d'acquisition, le statut et le sens sont très variables.

L'Université de Montpellier, réunissant entre autres, depuis janvier 2015 les facultés des sciences et de médecine, conserve des collections représentatives de cette diversité ${ }^{1}$. Quantitativement néanmoins, l'ensemble constitué pour l'étude de l'anatomie est le plus important et sera abordé ici plus particulièrement.

\section{La création du conservatoire d'anatomie}

La collection anatomique montpelliéraine est abritée aujourd'hui dans la galerie édifiée à cette fin en 1851 par l'architecte Pierre-Charles Abric (1800-1871) : le conservatoire d'anatomie présente, dans un cadre majestueux rythmé par des colonnades, et sous l'égide de médecins célèbres et d'allégories de diverses Sciences peints par l'artiste montpelliérain Jean-Pierre Montseret (1813-1888), une succession de vitrines déclinant la description du corps humain en détail ainsi que ses pathologies (fig. 1). Les débuts de cette collection sont cependant antérieurs à la présentation au sein de cette vaste galerie et datent de l'extrême fin du XVIII ${ }^{\mathrm{e}}$ siècle. Son premier noyau est en effet directement issu des réformes révolutionnaires.

Les idées liées à un nouveau mode d'enseignement de la médecine, développées dès le XVIII ${ }^{\mathrm{e}}$ siècle dans le contexte des Lumières, aboutirent à sa restructuration profonde pendant les dernières années de la Révolution. Cette 
rénovation de l'enseignement passa notamment par la constitution de collections anatomiques.

La loi du 18 août 1792, qui supprima les corporations et les associations, mit fin à l'organisation d'Ancien Régime : à Montpellier, l'Université de médecine et le collège des chirurgiens de Saint-Côme furent dissous. Néanmoins, la nécessité de former des médecins et chirurgiens, plus précisément des officiers de santé en période de guerre révolutionnaire, aboutit à la création, par un décret de la Convention nationale du 4 décembre 1794, de trois Écoles de Santé à Paris, Strasbourg et Montpellier². En 1795, Jean-Antoine Chaptal (1756-1832), nommé professeur de chimie appliquée aux arts et à l'industrie à l'École de Santé de Montpellier, fut chargé de sa réorganisation et l'installa dans l'ancien monastère-collège Saint-Benoît ${ }^{3}$, devenu à partir de la Renaissance palais épiscopal.

La réforme institutionnelle de la Convention, qui accomplit la réunion en un même lieu des futurs médecins et chirurgiens, s'accompagna d'une volonté d'améliorer l'enseignement en donnant une plus large place aux études pratiques : le décret prévoyait ainsi la création dans chacune de ces Écoles d'un conservatoire constitué d'un cabinet d'anatomie, d'instruments de chirurgie et d'une collection d'histoire naturelle médicale. Un conservateur, chargé de cette collection, devait par ailleurs effectuer des démonstrations des drogues et des instruments de chirurgie.

\section{Le corps humain, objet de pratique pédagogique}

La collection est logiquement centrée autour de l'étude de l'anatomie humaine, normale et pathologique. Ce thème principal est complété par des séries ayant trait à l'anatomie comparée et à la zoologie d'une part, à l'archéologie et à l'anthropologie physique d'autre part. Les pièces conservées sont soit artificielles, en cire, plâtre ou papier mâché, soit naturelles. Les restes humains représentent ainsi presque la moitié de la collection et revêtent différents aspects : préparations anatomiques sèches ou en fluides, ostéologie, coupes histologiques ${ }^{4}$.

La galerie, d'une superficie de $500 \mathrm{~m}^{2}$, est constituée de seize armoires murales vitrées conçues dès l'origine, de vingtdeux vitrines-tables et de quelques vitrines complémentaires ajoutées par la suite pour accompagner l'accroissement de la collection. Malgré un désordre apparent dû à l'accumulation de nombreuses pièces dans les vitrines, et à certains déplacements ou ajouts d'objets parfois peu cohérents au sein d'une même vitrine, la collection suit une classification globalement méthodique, liée à son propos pédagogique. Paul Gilis (18571929), conservateur entre 1917 et 1927, opéra une réorganisation fondamentale des collections à laquelle correspond pour grande partie l'état actuel de présentation ${ }^{5}$.

Lors de la création du conservatoire à la fin du XVIII ${ }^{\mathrm{e}}$ siècle, la collection était quasiment inexistante et les conservateurs successifs veillèrent à son enrichissement progressif ${ }^{6}$. Un premier lot d'une trentaine de pièces fut acquis lors de la succession de Philippe-Laurent de Joubert (1729-1792), le 4 fructidor An V (21 août 1797) ${ }^{7}$. Aux modalités classiques d'acquisition, par achat ou don, s'ajoute ici une spécificité du conservatoire d'anatomie : par essence lié à l'enseignement, le conservatoire reçut des pièces réalisées dans le cadre de cette activité. Ainsi, dès le 4 brumaire An VII (25 octobre 1798), l'École décida que les étudiants souhaitant s'inscrire aux examens terminaux devaient réaliser une pièce d'anatomie ; ces pièces furent alors déposées au conservatoire et consignées dans le premier registre des collections, entre l'An VII et l'An X (1798-1802) ${ }^{8}$. Néanmoins, si 77 numéros liés à cette décision de l'École apparaissent dans le registre, une dizaine seulement concerne en fait l'anatomie humaine réelle, les autres pièces étant relatives à l'anatomie humaine artificielle, à l'anatomie comparée, à l'instrumentation ou encore aux préparations pharmaceutiques. En outre, dès l'An VII (1798), apparurent les premiers dépôts de pièces anatomiques par les professeurs et, l'année suivante, par le prosecteur Bernard Delmas.

C'est en effet le corps enseignant qui, en toute logique, participa majoritairement à l'enrichissement du conservatoire : non seulement les professeurs d'anatomie, mais aussi leur
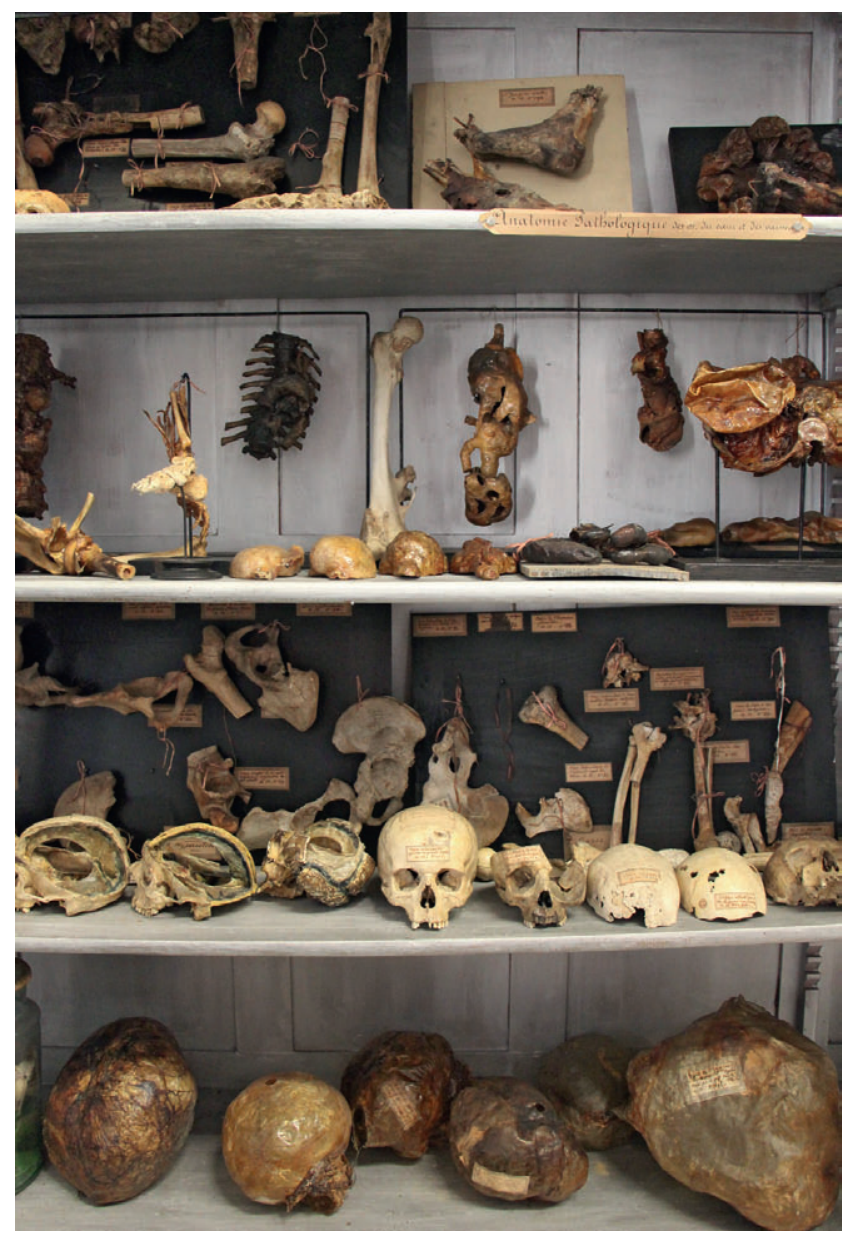

Fig. 2. Vitrine murale 8, ostéologie pathologique. (c) Université de Montpellier. 
équipe d'encadrement constituée de prosecteurs dès 1794 , puis de chefs de travaux anatomiques à partir de 1803. L'enseignement de l'anatomie, en partie privé jusque dans les années 1830, s'institutionnalisa progressivement à partir de la création de l'École pratique d'anatomie et de chirurgie en 1811, destinée à compenser le déséquilibre entre le nombre d'étudiants et le nombre de cadavres que l'École de Santé pouvait se procurer pour les dissections. Deux postes d'aides d'anatomie furent créés pour aider au fonctionnement de cette École ${ }^{9}$. Les concours de chef de travaux anatomiques, de prosectorat et d'adjuvat engendrèrent une émulation considérable entre les candidats qui devaient produire plusieurs dizaines de pièces d'anatomie (humaine et comparée) afin d'être évalués. Par exemple, lors du concours pour le poste de chef des travaux anatomiques de 1851, il n'y eut pas moins de cent trente-neuf pièces d'anatomie humaine préparées par les quatre candidats, outre les cent vingt-deux pièces d'anatomie comparée ${ }^{10}$. Les candidats étaient jugés bien sûr sur la qualité des dissections, mais aussi progressivement sur celle de la préparation, avec le développement de nouvelles techniques de conservation. Le professeur Joseph Grynfeltt (1840-1913), conservateur et auteur d'une notice assez détaillée sur le conservatoire, se réjouit ainsi : «Signalons seulement l'excellente voie dans laquelle vient d'entrer le jury du dernier concours pour le prosectorat [1878], en demandant aux candidats d'exécuter leurs préparations suivant le procédé de Laskowski, qui permet de conserver aux parties molles leur souplesse et qui, par suite, prévient la détérioration rapide des pièces résultant de leur dessiccation, tout en rendant leur étude plus fructueuse et plus commode ${ }^{11}$. " Nombre des candidats reçus aux postes de chefs de travaux et de prosecteurs firent par la suite une brillante carrière universitaire ${ }^{12}$.

Ces multiples pièces d'anatomie issues des concours sont ainsi fort représentées au sein des vitrines du conservatoire. Ce dernier a également bénéficié de nombreux dons de chirurgiens, professeurs d'anatomie, de pathologie ou de clinique. Une série de pièces provient ainsi des dons de Jacques-Mathieu Delpech (1777-1832) : elles présentent des difformités osseuses, relatives notamment à la colonne vertébrale, qui ont été au cœur des recherches de ce chirurgien et professeur de clinique externe (fig. 2) ${ }^{13}$. Ces pièces illustrent l'intérêt croissant pour l'anatomie pathologique à partir des années $1820^{14}$. Plus tard, ce fut le professeur Jules Mouret (1865-1928) qui donna un ensemble d'os et de préparations anatomiques relatifs au crâne, rehaussés de couleurs pour souligner les différents rapports osseux, vasculaires et nerveux. Cette série correspond à la discipline qu'il enseigna entre 1920 et 1928, l'oto-rhinolaryngologie (fig. 3) ${ }^{15}$. Il convient de noter également un lot

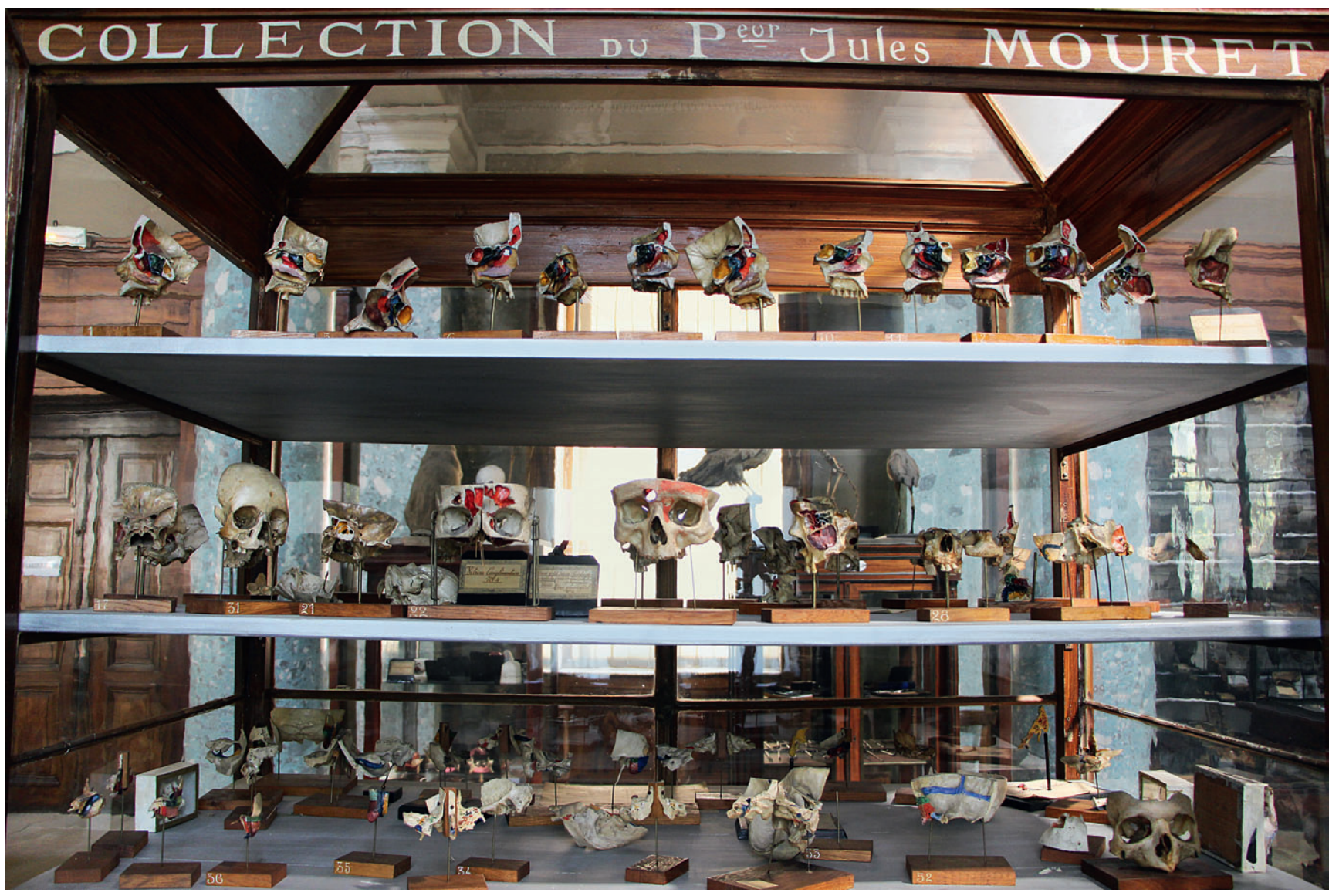

Fig. 3. Vitrine complémentaire 4, collection du professeur Jules Mouret.

(c) Université de Montpellier. 


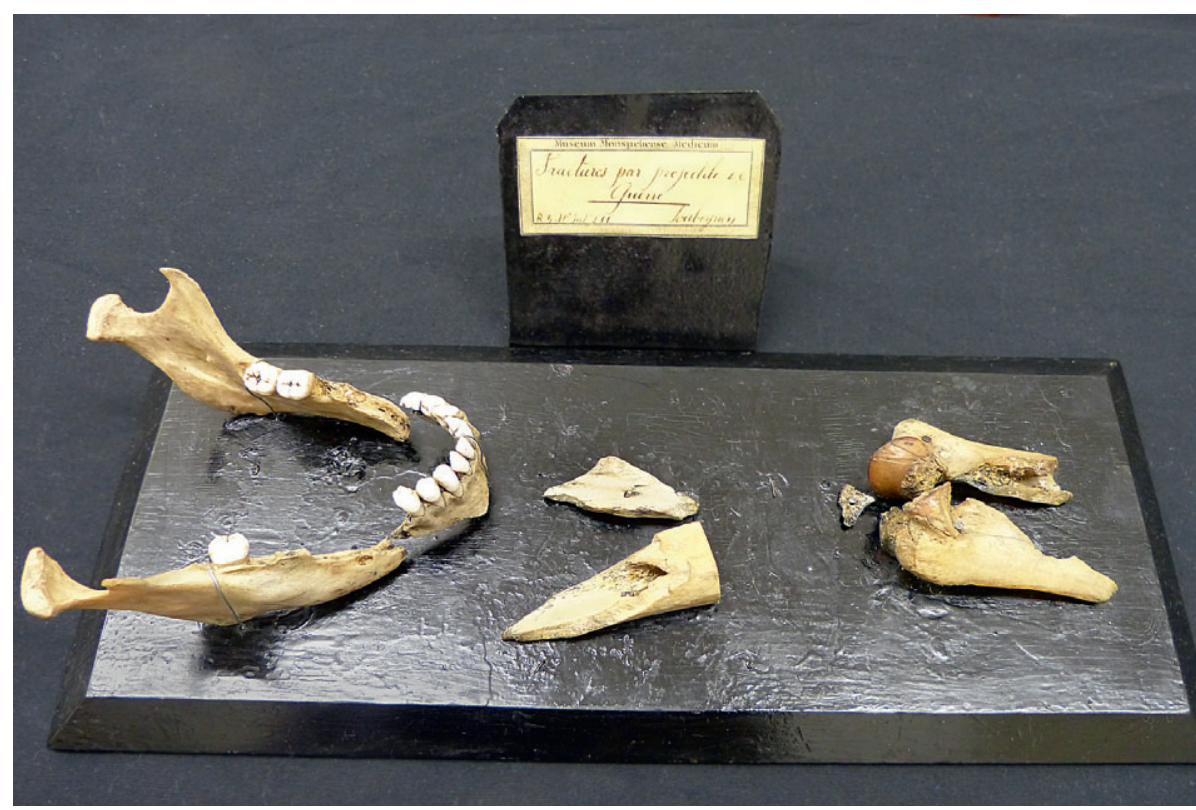

Fig. 4. Vitrine-table 18, fracture par projectile de guerre, Don M. Soubeyran. (c) L. Cadot.

constitué dans le contexte particulier de la Première Guerre mondiale : le professeur Paul Soubeyran qui fut chirurgien sur le front rapporta une série de fragments osseux qui sont un témoignage bouleversant de la gravité des blessures causées par les éclats d'obus (fig. 4) ${ }^{16}$. Un dernier ensemble important compléta encore le conservatoire au début du $\mathrm{Xx}^{\mathrm{e}}$ siècle : entre 1927 et 1952, le professeur d'anatomie Jean Delmas (1882-1966) géra la collection et l'enrichit de nombreuses pièces, notamment d'une série de coupes pratiquées sur le sujet congelé qui, accompagnées de planches légendées, forment un ensemble pédagogique remarquable à une époque où les techniques d'imagerie médicale n'existaient pas ${ }^{17}$.

\section{Extension du domaine de l'anatomie}

Certains ensembles de restes humains ne concernent pas directement l'anatomie, mais ont néanmoins été donnés au conservatoire, considéré en quelque sorte comme un musée traitant des sciences de l'homme dans leur globalité.

Un grand nombre de pièces a ainsi pour origine l'action décisive de Joseph-Marie Dubrueil (1790-1852), professeur d'anatomie de 1824 à sa mort. Non seulement il fournit à plusieurs reprises pendant sa période d'exercice des pièces, mais, à son décès, le conservatoire bénéficia en outre du don de sa collection par sa famille. Elle a non seulement trait à l'anatomie pathologique et comparée, mais comprend de surcroît une série considérable de crânes et têtes de peuples du monde entier (fig. 5) ; ce dernier ensemble est bien sûr à replacer dans le contexte de l'anthropologie physique telle qu'elle se concevait au XIX ${ }^{\mathrm{e}}$ siècle $^{18}$. Il est par ailleurs complété par quelques éléments rapportés de voyages extra-européens, par le botaniste Alire Raffeneau-Delile (1778-1850), qui participa avec Bonaparte à la campagne d'Égypte ${ }^{19}$, ou plus tard par le professeur Charles Martins (1806-1889), naturaliste et directeur du Jardin des Plantes, qui donna beaucoup au conservatoire $^{20}$.

Parmi les pièces ne relevant pas de l'anatomie, il faut encore noter plusieurs pièces ou lots relatifs à l'archéologie. Le conservatoire abrite ainsi une dizaine de momies entières, dont deux guanches, qui font partie des premières acquisitions $^{21}$, et huit issues des fouilles de la nécropole égyptienne d'Antinoé, reçues par trois envois successifs de l'État entre 1901 et $1907^{22}$. Les dons et dépôts de matériel archéologique antinoïte aux musées d'universités furent destinés aux facultés de lettres et de médecine dans un but pédagogique. Les momies du conservatoire d'anatomie pouvaient certes être considérées comme des objets de curiosité, dans la lignée des Wunderkammer, mais aussi des moyens d'observation du corps humain et d'archéologie de la médecine, comme l'illustre l'une des momies de l'envoi de 1907 qui présente un dispositif orthopédique ${ }^{23}$. Un autre ensemble, plus local, provient de fouilles archéologiques réalisées au début du $\mathrm{XX}^{\mathrm{e}}$ siècle dans deux grottes de la Lozère et du Gard par les docteurs André et Marc Romieu ${ }^{24}$. Le don au conservatoire s'explique ici logiquement par la formation médicale de ces deux archéologues ; il confirme en outre la nature plurielle de la collection qui, quoique majoritairement consacrée à la pure anatomie, s'ouvre vers d'autres champs d'étude de l'homme. 
La collection du conservatoire d'anatomie de la faculté de médecine de Montpellier est un témoignage remarquable de l'histoire et des pratiques de l'enseignement de l'anatomie au XIX ${ }^{\mathrm{e}}$ et au début du XX ${ }^{\mathrm{e}}$ siècle, avant le développement des technologies nouvelles d'investigation du corps humain et l'avènement de l'anatomie du vivant. Il convient donc de souligner la destination intrinsèquement pédagogique de cet ensemble, et le lien fort qu'il entretient avec la recherche et l'enseignement de son époque. La constitution et l'enrichissement de cette collection, de manière collective, par les étudiants et les enseignants, et pour les étudiants, ont par ailleurs probablement contribué à renforcer l'identité de la communauté des médecins montpelliérains. De nos jours, cet ensemble a certes perdu sa valeur d'usage première, mais conserve une valeur patrimoniale exceptionnelle : les étudiants en médecine auxquels il était destiné n'y passent plus qu'occasionnellement ; en revanche, le conservatoire d'anatomie attire désormais un public plus large d'adultes, d'étudiants en sciences humaines ou en arts plastiques, ou encore de lycéens, tous intéressés par la dimension extrêmement originale de cette collection, qui est ainsi vue chaque année par plusieurs milliers de personnes lors des Journées européennes du Patrimoine.
Les anciens musées Delmas-Orfila-Rouvière, provenant de l'Université Paris V-Descartes et composés notamment de près de trois mille éléments humains, sont venus récemment encore accroître cette collection ${ }^{25}$. L'Université de Montpellier est désormais dotée d'un ensemble anatomique de premier plan, classé au titre des Monuments historiques, qui fait l'objet d'une réflexion associant l'Université et la DRAC Occitanie, afin d'en améliorer la conservation et la présentation au public. Dans l'attente du déploiement prochain de ces deux ensembles au sein du Bâtiment historique de la faculté de médecine, qui nécessitera en amont un chantier des collections et l'élaboration d'un projet muséographique, l'accent est actuellement mis, autant que possible, sur la médiation, assurée pour l'heure par les guides de l'Office du Tourisme de Montpellier, formés à cet effet, et par l'équipe de l'Université.

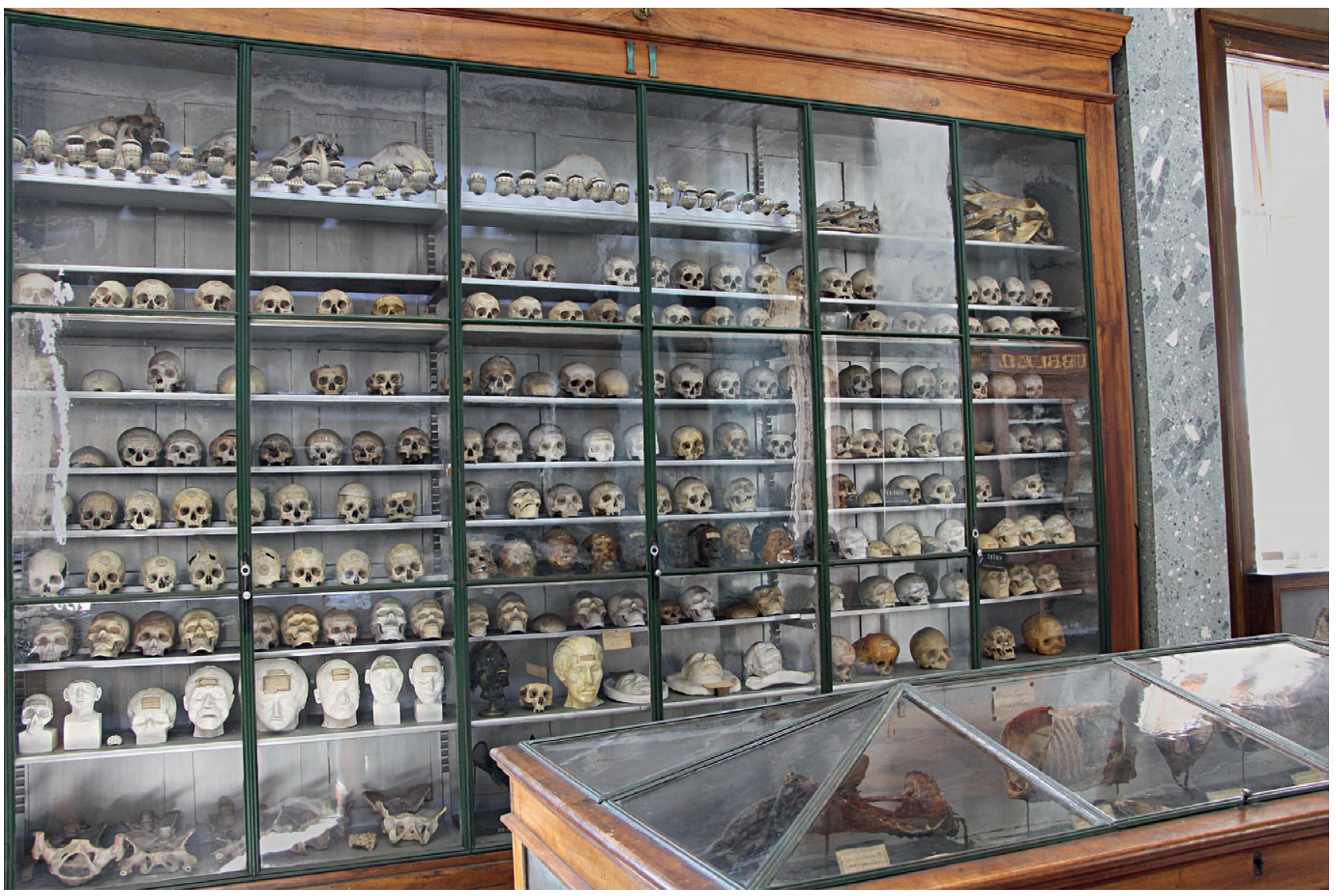

Fig. 5. Vitrine murale 11, anthropologie physique. ( Université de Montpellier. 


\section{Notes}

1. L'Université de Montpellier est issue de la fusion en janvier 2015 des anciennes Universités Montpellier 1 et Montpellier 2. Cette fusion a engendré une réorganisation administrative, et plus particulièrement la création d'une Direction de la culture scientifique et du patrimoine historique, en charge notamment des collections universitaires.

2. Lemire, 1990, p. 214-215 : le décret, porté par le chimiste et médecin AntoineFrançois de Fourcroy (1755-1809), et inspiré par les idées de Félix Vicq d’Azyr (1748-1794), prôna le développement de l'enseignement pratique en réaction à la routine de celui de l'Ancien Régime, qui ne comprenait pas les sciences physiques et exactes, ni l'expérience au lit des malades. Il prévoyait huit professeurs à Montpellier ; les professeurs étaient nommés par le Comité d'Instruction publique et devaient avoir une activité de recherche. Chaque École comportait en outre un directeur, un bibliothécaire et un conservateur des collections, également chargés de cours.

3. Ministre de l'Intérieur sous le Consulat, Chaptal veilla encore au développement de l'École de Montpellier en la dotant d'un amphithéâtre d'anatomie, réalisé par l'architecte Claude-Mathieu Delagardette (1762-1805), et en favorisant l'acquisition d'un ensemble de cires anatomiques dues à Felice Fontana. Cf. Palouzié, 2010.

4. Les 2440 restes humains estimés, soit $43 \%$ de l'ensemble de la collection, sont composés pour l'essentiel de préparations anatomiques sèches $(72 \%$, dont $1 \%$ de momies). Les autres types se répartissent comme suit : ostéologie (18\%, dont $5 \%$ liés à la collection anthropologique de crânes), pièces en fluides $(10 \%$, dont $3 \%$ liés à la collection de tératologie). Les coupes histologiques sont peu nombreuses au conservatoire.

5. La fonction de conservateur s'éteignit après le départ de Paul Gilis et le conservatoire d'anatomie fut rattaché au laboratoire d'anatomie.

6. Les pièces et objets du conservatoire d'anatomie sont inscrits dans neuf registres, allant de l'An VI à 1926, qui ont été complétés par les conservateurs successifs et sont actuellement conservés dans le fonds des archives anciennes à la Bibliothèque universitaire de la Faculté de médecine. Ces registres ne suivent pas à proprement parler une numérotation " réglementaire » : la numérotation reprend en effet entre chaque catégorie, ou à chaque nouveau registre, ce qui correspondrait à un état des lieux de la collection récolée à un moment donné, ou encore entre chaque vitrine, selon les périodes et le choix du conservateur. Malgré les difficultés engendrées par cette numérotation multiple, les registres sont une source précieuse pour documenter la collection, en raison de certaines descriptions de pièces mentionnant leur origine, ou la date de leur dépôt.

7. Registre du conservatoire $n^{\circ} 1$, p. 5 . Seigneur du Bosc, baron de Sommières et de
Montredon, Philippe-Laurent Joubert fut président à la cour des comptes, aides et finances de Montpellier et trésorier des États du Languedoc. Naturaliste, il constitua un riche cabinet d'histoire naturelle, et fut également co-fondateur de la Société des beaux-arts de Montpellier en 1779.

8. Registre $n^{\circ} 1$, p. 7-16.

9. Bonnel, 2010, p. 14 ; Dulieu, t. 4-1, p. 310-315 : le règlement de cette École instituait que dix étudiants en anatomie et dix étudiants en chirurgie pouvaient s'initier aux dissections, ainsi qu'à des rudiments de chirurgie. Leur présence était requise quatre heures par jour entre novembre et avril ; ils étaient recrutés par concours et leur stage durait deux ans.

10. Registre 3, passim.

11. Grynfeltt, 1879, p. 23.

12. Dulieu L., ibid. : le nombre croissant d'élèves de l'École pratique, ce qui confirme son succès, ainsi que l'inadaptation des locaux destinés à l'enseignement pratique l'ancienne sacristie de la cathédrale adjacente et la dernière aile du cloître - eurent pour conséquence la construction en 1868 d'un pavillon d'anatomie. Ce dernier fut détruit et remplacé par un édifice nouveau sous le décanat de Gaston Giraud (1941-1960).

13. Delpech publia ainsi en 1828 un ouvrage lié à ses recherches intitulé $D e$ l'Orthomorphie, par rapport à l'espèce humaine : ou recherches anatomico-pathologiques sur les causes, les moyens de prévenir, ceux de guérir les principales difformités et sur les véritables fondemens de l'art appelé orthopédique.

14. Grynfeltt, 1879, p. 10.

15. L'otologie, la rhinologie et la laryngologie ne furent réunies qu'à l'extrême fin du XIX ${ }^{e}$ siècle et au début du XX ${ }^{\mathrm{e}}$. L'anatomiste Jules Mouret en fut le fondateur à Montpellier. Cf. Mouret J., « De l'utilité d'un enseignement d'oto-rhino-laryngologie », Montpellier médical, t. XXIV, 1907.

16. Registre $n^{\circ} 5$ ter, p. 17-19:

29/12/1921, pièces de blessures de guerre recueillies aux armées, 1914-1918, Ambulance 6/13, Autochir n ${ }^{\circ} 37$. Don M. Soubeyran. Cf. Exp., Médecine au champ d'honneur, 2014.

17. Laux, 1958, p. 129 ; Delmas, 1938, p. 26 : Paul Delmas indique que les collections ont été réorganisées par le professeur Jean Delmas. Il s'agit sans doute de l'aménagement des vitrines où sont présentées ces coupes.

18. Registre ${ }^{\circ} 3$, p. $84: n^{\circ}$ 187-267 : don du 3 septembre 1854, par la famille de feu le professeur Dubrueil ; nº 187-190 : quatre têtes préparées par voie de dessiccation ; $\mathrm{n}^{\circ} 191$ : une tête [crâne] trouvée dans les ruines d'Athènes ; n 192-193 : deux têtes [crânes] d'hydrocéphales ; n 194-256: 63 têtes [crânes] ; n 257-266 : 9 têtes [crânes] d'anatomie pathologique ; ${ }^{\circ} 267$ : ensemble d'ostéologie pathologique. Cf. Jean, 2015, 9 p. Il faut noter qu'une tête Maori tatouée, dont un moulage en plâtre polychrome est également conservé, a été restituée en 2012.

19. Registre $n^{\circ} 3, n^{\circ} 352$ et 353 : têtes de momies (homme et femme) provenant des collections de M. le professeur Delille, données par M. [Jean-Joseph Bonaventure] Laurens, agent comptable de la faculté. Registre $\mathrm{n}^{\circ} 4$, p. 12 : armoire 2 (armoire 11 en 1917), $n^{\circ} 61$ et 62 : « têtes de momies
d'Égypte/prises par le professeur Delisle dans les pyramides d'Égypte».

20. Registre $\mathrm{n}^{\circ} 4$, p. $16: \mathrm{n}^{\circ} 216$, tête provenant des environs d'Adelaïde, don Pr. Martins, 1870.

21. Registre no 2 , p. $2: n^{\circ} 35$ et 36 , momies de Guanche, 15 prairial an XIV (4 juin 1806), apportées des Canaries et données par M. le professeur Auguste Broussonet.

22. Larquier (de), 2009, p. 29-32 ; il ne s'agit pas en réalité de momies embaumées, mais de corps naturellement desséchés dans le sable d'Antinoé, en raison du climat désertique (ibid., p. 5).

23. Larquier (de), 2009, p. 25.

24 . Vitrine 15 , les cartels anciens précisent : «Ossements humains provenant de la grotte sépulcrale de la Petite Suisse près Meyruès [sic] (Lozère) ; âge du bronze (-1000 av. J.-C.) » et « Ossements provenant de la grotte sépulcrale de Bramabiau, commune de St Sauveur des Pourcils (Gard) ; plusieurs crânes correspondant à ces squelettes sont déposés au Musée de Nîmes ; âge de la Pierre polie (-2000 av. J.-C.) ; race des BaumesChaudes (Cro-Magnon métissé) ».

25. Palouzié, Girard, 2015 ; Delmas, 1995.

\section{Bibliographie sélective}

Bonnel F., Claustre J.-E., Bonnel C., 2010, «L'enseignement de l'anatomie à Montpellier », Nunc Monspeliensis Hippocrates, $\mathrm{n}^{\circ} 10$, p. 6-20.

Delmas A. (dir.), 1995, « Musées d'anatomie Delmas-Orfila-Rouvière », Surgical and Radiologic Anatomy - Journal of Clinical Anatomy ( $\mathrm{n}^{\circ}$ spécial), Springer, $154 \mathrm{p}$.

Dulieu L., 1986-1990, La médecine à Montpellier, tome IV, Avignon : Presses universelles, 2 vol.

Grynfeltt E., 1879, Notice sur le conservatoire de la faculté de médecine de Montpellier Montpellier, $24 \mathrm{p}$.

Laux G., 1958, « Le musée anatomique de la Faculté de médecine de Montpellier et son histoire ", Montpellier médical, $3^{\mathrm{e}}$ série, t. XIV, no 1 , p. 126-130.

Lemire M., 1990, Artistes et mortels, Chabaud, $446 \mathrm{p}$.

Palouzié H., 2010, Felice Fontana, l'aventure des cires anatomiques de Florence à Montpellier, DRAC-LR, collection « Duo », $50 \mathrm{p}$.

Palouzié H., Girard C., 2015, «La réunion des collections des universités de Montpellier et Paris ", Chantiers, $n^{\circ}$ 9, p. 18-21.

Exp., Montpellier, 2014 : Médecine au champ d'honneur. Des hommes et des avancées médicales, [Faculté de médecine, Université Montpellier 1, 2014], 60 p.

\section{Documents inédits}

Jean S., 2015, Le don de Toi Moko à l'Université de Montpellier, dossier de recherche (non publié), $9 \mathrm{p}$.

Larquier (de) N., 2009, Momies et tissus coptes de Montpellier : un siècle de mésaventures, Mémoire École du Louvre (non publié), 2 vol. 